\title{
Dificultades rítmicas en la lectura musical visual durante los primeros años del aprendizaje de piano
}

\section{Béatrice Dubost}

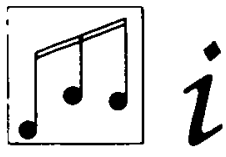

El papel de la codificación escrita en el uso de un instrumento musical es esencial para ir más allá de una competencia muy limitada. La lectura de la notación musical es una competencia que incluye aspectos que trascienden la simple decodificación tonal o una serie de instrucciones para la ejecución en un instrumento. Este artículo explora el repertorio de procesos psicológicos (perceptivos, representacionales, motores) que permiten construir una buena alfabetización musical.

\section{INTRODUCCION}

La lectura musical ocupa un lugar no desdeñable en el aprendizaje pianístico, tanto para los principiantes como para los virtuosos, ya que el músico a menos que se sepa de memoria los fragmentos de un repertorio o que esté improvisando, se encuentra con frecuencia en la situación de leer o releer textos musicales.

Incluso en el caso de que ya haya oído una determinada obra, que reconocerá al oírla, así como su forma, título y autor, si nunca ha visto la partitura escrita que en ese momento tiene ante sus ojos la tendrá que descifrar, y deberá poder hacerlo con exactitud y precisión si se trata de acompañar repentizando a un determinado instrumento o cantante. Para ello deberá seguir un proceso complejo. Pasemos a revisar los aspectos generales previos a las experiencias relacionadas con la lectura visual melódico-rítmica que vamos a presentar en este trabajo.

La percepción de la notación musical en situación de lectura pianística

Los símbolos de la notación musical no son únicamente una serie de instrucciones para la ejecución (salvo en determinadas formas de música con- 
temporánea), sino que engloban también aspectos de la estructura o significados de la música que no podrían estar presentes en una descripción puramente física de los sonidos (Nattiez, J. J., 1975). La partitura de música tonal explicita una relación de notas en medio de claves, medidas, alteraciones... y necesita que el lector las perciba, comprenda su significado (Francès, R., 1958).

Los motivos melódicos, rítmicos y armónicos que constituyen la organización del discurso musical tonal se superponen o se suceden en movimientos ajustados o desajustados, paralelos o contrarios, en frases marcadas con valores largos o con silencios antes de ser interpretadas de forma alternativa o simultánea a dos manos con su relieve expresivo. La melodía no siempre es la única secuencia expresiva de un fragmento, también lo son los acordes de acompañamiento que comportan en sí mismos una melodía, lo que hace entender que las notas forman parte de unos acordes pivote de las tonalidades, en los que se dará mayor importancia a los soportes de la modulación. Los motivos rítmicos participan de la melodía y de la armonía, las series armónicas contienen ritmos que incluyen cantos que hay que subrayar.

La lectura tiene una doble vertiente, horizontal y vertical inducida por la música y sus niveles de organización.

Dentro de las frases, el lector sigue el encadenamiento de tonalidades que varían con las modulaciones. Tiene en cuenta matices y frases que están indicadas y que acompañan a los valores de las notas que son los puntos de apoyo, las dinámicas móviles y vivas de la obra musical.

Si la lectura oral de los símbolos del lenguaje escrito tiene sus ritmos, sus acentos silábicos, de modificación, que dan sentido a frases insertas en una puntuación lineal cuya fisonomía gráfica es muy distinta de la de la notación musical, el descifrado de un fragmento de piano está sometido a indicaciones de tempo, mientras que en la lectura oral no se da esta misma exigencia.

Teniendo en cuenta la alternancia de pasajes sencillos y más complicados, pasajes lentos y rápidos, puede ocurrir que el tempo del comienzo, impuesto o elegido, no se pueda conservar durante todo el fragmento, dando lugar a que éste se ralentice o se detenga.

Si tocar muy lento o muy rápido puede contribuir a deformar o anular una característica de la obra, permanecer atado estrictamente a su mensaje significaría que la música sólo tendría significado para el oyente según una forma precisa y justa de interpretación, cercana al texto y a sus indicaciones estructurales y estilísticas.

No todos los pianistas, ni siquiera aquéllos con una gran experiencia, consiguen descifrar la música con la misma facilidad. Todos los niveles son posibles, desde los que leen con dificultad a los que hacen una lectura fluida.

A la luz de las investigaciones llevadas a cabo en psicología de la percepción, se sabe que los lectores hábiles y rápidos fijan su mirada en dos puntos estratégicos del texto, a partir de los cuales se puede prever lo que va a seguir, estableciéndose una relación entre el umbral de memoria inmediata y la rapidez de lectura. (Según Sloboda, los mejores lectores tendrían un umbral de 6 a 5 notas y los peores de 3 a 5.) Las fijaciones serían más numerosas en los pasajes difíciles, de notas alteradas, de ritmos complejos con encadenamientos poco previsibles. Por el contrario, cuando las 
gamas de arpegios o los distintos motivos nos evocan formas conocidas, la lectura anticipa con más facilidad y la mirada economiza una lectura detallada para retener únicamente la configuración global, avanzando y pasando a las indicaciones siguientes (Weaver, 1943).

La posibilidad de decidir la probable continuación de la frase representa una ventaja para la lectura y su rapidez, ello implica un conocimiento explícito o implícito de las reglas de la armonía y del ritmo (Sloboda, 1985) y la capacidad de transcribir este conocimiento en los movimientos necesarios para producir los sonidos y los valores apropiados.

\section{Las manos, el conocimiento del teclado y la anticipación motora}

La utilización de mandos manuales en la transposición visioespacial de sonidos, de valores rítmicos de tiempos, de agrupaciones armónicas más o menos complejas en el espacio del teclado, es un aspecto específico de la lectura de la música instrumental.

La interpretación pianística, que no se define únicamente como conducta lúdica física y mental, sino que, como forma de interpretar, implica la cualidad artística del ejercicio de dirección necesario para asegurar la adecuación viso-motriz entre los dos espacios visuales (partitura y teclado) de la que surge la imagen sonora de la música.

El teclado se distribuye entre las dos manos que lo van conociendo, se orientan en los obstáculos topográficos de su relieve, exploran el espacio, aprecian las dimensiones de los intervalos y de los recorridos. Manteniendo su independencia y libertad, las manos participan de un mismo impulso, tocando en suplencia o en convergencia, se intercambian, se reparten las dominancias de las voces melódico-rítmicas o las acompañan. Las manos se acercan o se alejan, se cruzan o se montan, se asocian o alternan en el espacio-tiempo discontinuo de los saltos, de las desviaciones. Se afirman simultáneamente para intercambiarse las sonoridades en sinergia o en relevo, son flexibles y adaptables al texto en las pulsaciones de urgencia y de primera necesidad al servicio de la comodidad inmediata.

El cerebro, que controla los gestos, lleva a cabo el ajuste de las tensiones y distensiones.

Las obras jerarquizan las funciones según den prioridad a la amplitud de movimientos o a las capacidades de manipulación precisa. Para controlar el gesto de desplazamiento rápido de un punto a otro, el cerebro debe tener una representación anticipada del espacio a recorrer (Paillard, 1985), una especie de «mapa interior» del teclado (B. Ott, 1978). Si los desplazamientos deben hacerse de un punto a otro del teclado en tal o cual dirección, con tal o cual intención expresiva, como en un juego de pelota, es necesario estar en el lugar y momento adecuados ya que el desarrollo de la música en el espacio-tiempo impone el respeto de las exigencias de ritmo y de tempo.

El ojo informa al cerebro, que activa su gesto mientras prepara el siguiente para la próxima trayectoria espacial. Para reconstruir la pieza musical en su novedad inmediata, el cerebro efectúa varias operaciones a la vez, que forman parte de una tarea global tanto más complicada si se tiene en cuenta que cada mano debe jugar con orientaciones, ritmos, dinámicas diferentes (Shaffer, L. H.). 
El sujeto recibe información que le viene de canales perceptivos diferentes, visuales, auditivos, táctiles, y establece correspondencias entre estas informaciones para reconocerlas y ajustarlas unas a otras (Hatwell, 1986). El tiempo de tratamiento de las informaciones a un nivel superior del aparato psíquico depende no sólo de las características del material a leer, sino de la rapidez cerebral para integrar los datos polisensoriales en un conjunto unificado y coherente (Smith, 1986).

El conjunto psíquico espacial y temporal que forman el espacio sonoro y el tiempo musical exige del lector una virtuosidad de la orientación, un dominio de sus variaciones sobre la base de un referencial de datos estables y bien establecidos que permiten la organización de la conducta pianística.

La habilidad de coordinación viso-audio-motora se basa en los recursos de una programación interna, adquirida mediante el aprendizaje relativo a los datos del propio lenguaje (Francès, 1958; Imberty, 1969; Zenatt, 1981) y a los que están relacionados con el conocimiento del teclado en el que se actualiza la música; esta estructura cognitiva, íntimamente enraizada en la memoria, entra en resonancia con los materiales de lectura musical cuando son reconocidos por el lector como estímulos familiares.

El paso correcto de la codificación a la percepción y después a la comprensión del lenguaje musical tonal plantea interrogantes sobre la importancia de los procesos de tratamiento de la información (Noizet, G., 1980) y de memoria (Lieury, A., 1981) en este tipo de situaciones.

\section{Aproximación exploratoria a las dificultades de lectura a través de experimentos sobre el descifrado}

En el nivel más bajo de lectura el sujeto muestra una gran lentitud. Los ojos están en constante movimiento de la partitura al teclado. El constante control visual de la postura de los dedos sobre las teclas introduce tales rupturas en el desarrollo de los pasajes musicales que éstos llegan a ser irreconocibles para el oyente.

Esta forma de descifrar recuerda los comienzos del aprendizaje, cuando la lectura se hace nota a nota, de forma errónea, vacilante, entrecortada, torpe, fragmentada, entrecortada de cortes, repeticiones, vueltas atrás, intentos de autocorrección.

El descifrado al piano se hace cada vez más fluido a medida que se desarrolla la técnica instrumental y se adquiere maestría con el progreso de un año a otro con el entrenamiento, pero se ha comprobado que en un nivel de estudios ya avanzado y sujetos que podrían ser buenos músicos en los que no se da este progreso.

Se plantea entonces la cuestión de saber dónde se producen los bloqueos:

- En el aprendizaje de la música, de su código singular, de sus ritmos, de su sintaxis armónica.

- En el aprendizaje instrumental en el que se asocia al desarrollo técnico de funciones motrices (mecánica digital) y perceptivas (información sonora y musical) el conocimiento del teclado.

- En el propio sujeto, en sus capacidades de percepción de integración, de situación en la producción instrumental de los datos de la música, 
de los valores de las notas, en tanto que éstas constituyen la forma temporal, que representan la forma en que la música se realiza.

El ritmo determina el paso del tiempo en su desglose de la duración, al repartir el orden y las proporciones da una conciencia exacta de la duración gracias a la pulsación que mantiene el reparto de los intervalos de tiempo en una periodicidad regular y fundamental. Si los acentos marcan la articulación de ritmos con las medidas dentro de las cuales figuran los motivos que las caracterizan, no se confunde con el sistema que le encierra.

La percepción del ritmo que lleva a su reconocimiento, se basa en lo que previamente se ha vivido y sentido en relación con el movimiento, dando vida al juego de lectura pianística que le anima, paralelamente al entrenamiento en la lectura vocal, hablada o cantada, que se lleva a cabo en las clases de solfeo.

Con el fin de explorar las capacidades rítmicas en la lectura visual tonal, hemos propuesto a jóvenes alumnos de conservatorio de diferentes grados de su aprendizaje, el descifrado de un texto melódico-rítmico de estructura binaria, escrito por necesidades del experimento que ahora describiremos, con sus modalidades y resultados.

\section{EXPERIMENTOS}

\section{Muestra}

El experimento se llevó a cabo con una muestra de 53 sujetos pertenecientes a varias escuelas de música. Los sujetos se repartieron en tres grupos de edad de 9 a 14 años y con tres niveles diferentes de piano (iniciación, preparatorio y elemental), lo que dio lugar a tres grupos compuestos de la siguiente forma:

$\begin{array}{lll}\text { Iniciación } & \mathrm{I} & \mathrm{N}=10: 9-10 \text { años } \\ \text { Repetidores } & \mathrm{I} & \mathrm{N}=10: 10 \text { años } 4 \text { meses }-11 \text { años } 8 \text { meses } \\ \text { Preparatorio } & \mathrm{P} 1 & \mathrm{~N}=10: 10 \text { años } 4 \text { meses }-11 \text { años } 8 \text { meses } \\ & \mathrm{P} 2 & \mathrm{~N}=6: 11 \text { años } 8 \text { meses }-12 \text { años } 2 \text { meses } \\ \text { Elemental } & \mathrm{E} 11 & \mathrm{~N}=7: 12 \text { años } 4 \text { meses }-13 \text { años } \\ & \text { E12 } & \mathrm{N}=10: 13 \text { años e meses }-14 \text { años } 6 \text { meses. }\end{array}$

Pruebas

\section{LECTURA MELODICO-RITMICA DE ESTRUCTURA BINARIA}

En esta prueba se presentó un texto melódico-rítmico escrito en 4 tiempos: (la unidad de tiempo estaba representada por la negra, la unidad de medida por la redonda). La clave de sol figuraba en el pentagrama superior. Las notas se sucedian de un pentagrama a otro en un movimiento melódico conjunto con el fin de limitar las dificultades rítmicas del teclado. Los ritmos que mantenían la melodía presentaban una dificultad creciente 
en la medida en que su desarrollo representaba una especie de barrido ocular horizontal del texto a pesar de los cambios de pentagrama. El paso de un pentagrama a otro se efectuaba después de un valor largo para dar al lector tiempo de leer más allá y prever el desplazamiento.

Las segmentaciones del texto después del cambio de clave correspondían a límites de frases que se respondían alternativamente.

La melodía era de caracter tonal. De do mayor modulaba al tono de sol (dominante). Por razones relacionadas con la forma de pasar la prueba, debimos cortar el texto, lo que explica que éste terminara sobre sol sin volver a la tónica «do».

\section{Procedimiento}

El texto melódico-rítmico propuesto era objeto de dos lecturas sucesivas, una vocal hablada y otra instrumental.

En la lectura vocal se pedía al sujeto que solfeara marcando la medida según el tiempo dado. La lectura vocal precedía siempre a la lectura instrumental.

El control del nivel de lectura vocal permitía:

- comprobar la evolución del dominio instrumental en función de la edad y el nivel;

FIGURA 1
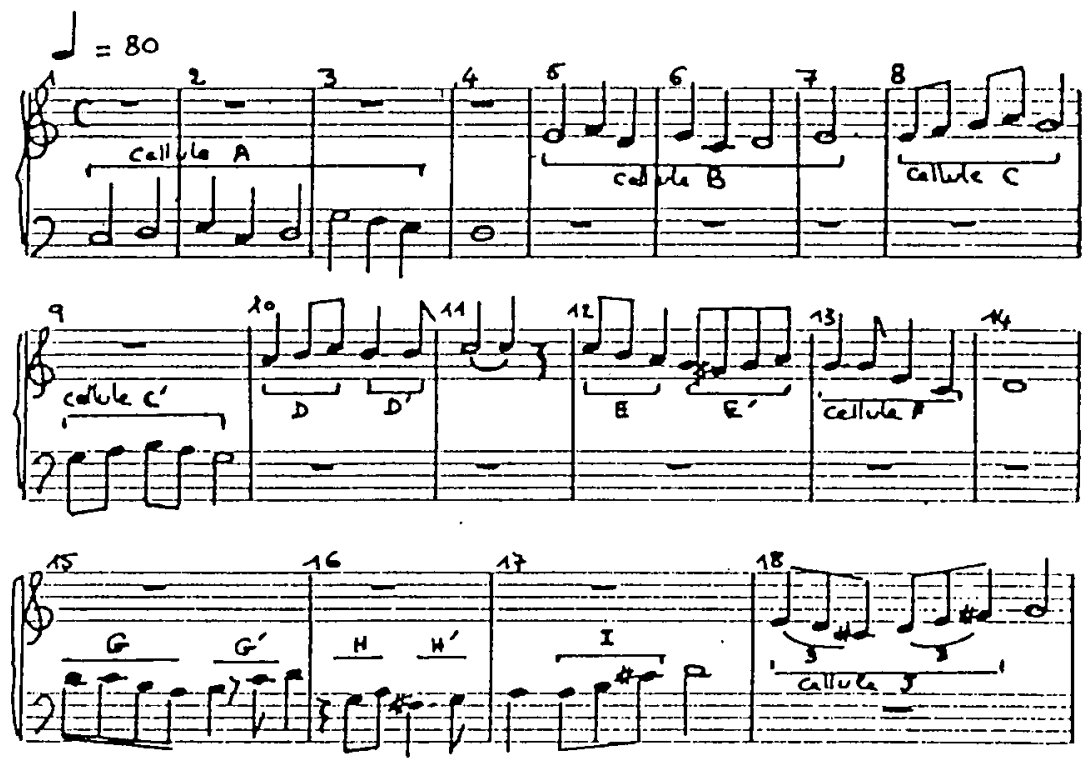

Lectura melódico-rítmica de estructura binaria.

- situar las adquisiciones de nociones en comparación con estas mismas adquisiciones cuando dependen del dominio de la técnica pianística;

- descubrir un determinado estado de coordinación viso-motora.

La observación de los logros se llevó a cabo mediante una recensión y contabilización de los errores de lectura según una tipología que permitía establecer unas formas de puntuación. 
Con el fin de proceder a la comprobación de los errores, las dos lecturas se retranscribieron en una sola versión vocal e instrumental, y más tarde se cronometró su duración.

Se estableció un primer grupo de resultados en función del número y el porcentaje de fallos en los sujetos de los diferentes grupos de nuestra muestra en cada uno de los grupos o células rítmicas aisladas para el análisis.

La presentación detallada de los aspectos formales de los errores tenía como finalidad la apertura de un campo de reflexión sobre las conductas psicológicas implicadas en la lectura visual musical.

\section{Tipología de los errores de lectura ${ }^{1}$}

Clasificamos y agrupamos los errores según las tres grandes categorías siguientes:

- errores de exactitud;

- errores relacionados con trabas en la «marcha de la lectura»

- tiempo de lectura.

\section{A. Errores de exactitud}

- Confusión de claves: (cclave), una nota en clave de sol leída en clave de fa o al revés;

- errores de notas: (EN), nota no leída en su clave original ni en otra clave, se le da otro nombre;

- errores de alteración: (Alt) un sostenido confundido con un bemol o un becuadro, o bien nota leída sin su alteración;

- errores de ritmo: (Rit), deformación de las estructuras rítmicas;

- olvidos: (o), notas, alteraciones, silencios...;

- errores de octavas y de registro (oct. reg), sólo aparecen en la lectura al piano: una nota bien identificada, pero tocada una, dos o tres octavas más alto o más bajo.

\section{B. Dificultades en la «marcha de la lectura»}

En ellas se reagrupó todo aquello que hacía más lento en el tiempo el desarrollo de la lectura.

- Las vacilaciones (vac), el sujeto tiene un momento de dificultad: sol... re, luego continúa su lectura;

- las repeticiones (repet), el sujeto repite una o varias veces la misma nota antes de pasar a las siguientes;

- las detenciones (D), la lectura se detiene, la acción se suspende durante un tiempo variable, desde unos segundos a varios minutos;

- las vueltas atrás (vuel), el sujeto vuelve atrás, y retoma el fragmento en 2, 3 ó 4 notas (a veces más) que acaba de leer o tocar. Se manifiestan con más frecuencia al comienzo de la lectura, pero también pueden aparecer en su trascurso;

- las vueltas atrás con autocorrección correcta $(\mathrm{V}+)$, el sujeto corrige instantáneamente un error cometido o no intenta volver atrás;

- las vueltas atrás con autocorrección incorrecta (V-), el sujeto vuelve atrás pero fracasa total o parcialmente en su autocorrección. 


\section{El tiempo de lectura}

Implica lentitud o exceso de rapidez, las irregularidades, y constituye la desviación del tempo dado a cada una de las lecturas.

\section{Resultados del experimento según la tipología}

Hemos agrupado los errores según las tres categorías, exactitud, dificultades y tiempo.

COMENTARIO: LECTURA MELODICO RITMICA DE ESTRUCTURA BINARIA (54 segundos)

Sólo el grupo EL2 mejoró su puntuación en exactitud en lectura oral en la lectura pianistica, las medias de trabas disminuyeron en todos los grupos.

Los grupos I, I', P1 y P2 mejoraron ligeramente su tiempo de lectura, pero sólo el grupo EL1 leyó en el tiempo establecido.

En conclusión, la presencia del ritmo en un texto melódico facilita la «marcha de la lecturan: las medias de dificultades disminuyeron para el conjunto de la muestra, que mejoró también sus logros en las categorías de exactitud y tiempos.

Los grupos de iniciación tuvieron dificultades en la identificación y reconocimiento de los ritmos ya en su lectura vocal. El grupo I' fue el más flojo.

Los sujetos del grupo EL2 no superaron el nivel de los sujetos del grupo P2, que se mostraron más rápidos en la lectura.

\section{Resultados del experimento en función del número de sujetos con errores en las células melódico-rítmicas del texto propuesto}

Cada célula rítmica aislada se ha representado con una letra en la Figura 1 .

\section{COMENTARIO}

Si tomamos el texto de la lectura melódico-rítmica binaria de la Figura 1, vemos que comienza con la clave de fa. Las cuatro primeras medidas encuentran su réplica en clave de sol. El cambio de clave se lleva a cabo después de un valor largo (redonda), lo que da tiempo a los lectores para avanzar en la lectura.

La célula $A$ en clave de fa que aparece en las tres primeras medidas provocó el mayor número de errores al piano en los niños de iniciación, estos errores fueron menos numerosos en la célula $B$ en clave de sol que aparece en las medidas 5,6 y 7 . Los errores disminuyeron en los de elemental de una forma de lectura a otra. El grupo de elemental leyó sin errores la célula B.

En la medida 8 del texto (célula C), las corcheas seguidas de una blanca en clave de sol se encadenan sobre su homóloga en clave de fa (célula C'medida 9). El valor largo (blanca) seguida de un tiempo de pausa (1/2 pausa) exige del lector el mantenimiento de la pulsación de la medida 7 a 8 , mientras pasa la línea y cambia de pentagrama, de la medida 8 a la 9.

Los errores en las células $C$ y C', comprendidas en estas dos medidas, son muy numerosos. Los resultados mejoran con el nivel, pero en el grupo elemental 6 sujetos de 17 leyeron el pasaje de forma errónea.

La comparación de las células $\mathrm{D} 2 \mathrm{E}$ (medidas 10 y 12) muestra que la célula D provoca más errores que la célula $E$. El paso del «sol» en clave de fa (medida 9), al «la» en clave de sol (medida 10) hace más difícil (en función del intervalo de $11 .^{2}$ ) la lectura de la célula $\mathrm{D}(\mathrm{J} /)$ que la de la célula $\left.\mathrm{E}(\Omega\lrcorner\right)$. La célula $\mathrm{E}$ viene después del tiempo de respiración dado por la blanca ligada a la negra seguida de una pausa (medida 11 ).

El porcentaje de errores de E + E' (medida 12) es superior al de D + D' (medida 10).

La medida 12, que reúne las dos células $\mathrm{E}+\mathrm{E}$ ', incitaba a los sujetos a establecer un equilibrio mejor al restablecer un valor largo sobre el tercer tiempo. La desestabilización de la lectura determinada por la forma de escritura en este lugar repercute en la medida saiguiente. 
Si se comparan las células F (medida 13), D (medida 10) y H' (medida 16), se comprueba que una misma fórmula rítmica en diferentes posiciones de un texto $y$ en diferentes claves, se lee de forma diferente.

Las dobles corcheas de la medida 15 (célula $G$ ) van después de un valor largo, recordando el modelo anterior a la aparición de las 4 corcheas de la medida 8 que sucedía a la blanca y la media pausa de la medida 7 . La pérdida de ritmo en este lugar fue frecuente, sobre todo en los sujetos de iniciación y preparatorio. Los de elemental mejoraron notablemente su puntuación en la lectura pianística.

En los tres grupos de nivel, las células $\mathrm{H}$ y H' de la medida 16 presentaron dificultades relacionadas con el silencio. Las semicorcheas resultaron más difíciles de leer para los sujetos de iniciación: la célula J (medida 18) dio lugar a más errores que la célula I (medida 17).

Si situáramos el criterio de éxito en un $75 \%$, es decir, con un porcentaje de errores de un $25 \%$, para decir que la prueba se había pasado con éxito, las nueve primeras medidas del texto se habrían pasado con éxito en los tres grupos en la lectura vocal; los valores rítmicos que componen estas medidas se adquirieron con el solfeo.

El aumento de los errores al piano en las células C y C' (medidas 8 y 9) demuestra claramente la distancia existente entre el conocimiento solfeístico del ritmo y su realización al piano.

A partir de la medida 10 el texto se hacía más difícil para los alumnos de iniciación, era todavía accesible en el nivel de preparatorio, y se podia considerar que la lectura se pasó con éxito en el nivel elemental en la modalidad vocal. Si es cierto que en este grupo de nivel la célula $G$, medida 15 , provocó un porcentaje de errores superior a un $25 \%$ en la lectura vocal, la disminución del número de errores en la lectura pianística demuestra que los valores de dobles corcheas se integra de una forma de lectura a la otra por efecto del aprendizaje. En el nivel elemental un $47 \%$ de los sujetos pasó con éxito la célula $\mathrm{H}+\mathrm{H}^{\prime}$ (medida 16) en las dos modalidades de lectura.

$\mathrm{El}$ análisis del conjunto de los resultados demostró que los errores no se recubren de una forma de lectura a otra o sólo lo hacen parcialmente. No son siempre los mismos sujetos los que cometen los mismos errores en la lectura vocal y al piano: unos se corrigen, otros hacen su aparición.

El aspecto de los errores variaba y sus formas evolucionaban de un nivel a otro. Los errores eran lábiles, móviles, ponían en evidencia diferencias en los sujetos, incluso en la forma de equivocarse, que ilustraremos con ayuda de ejemplos.

\section{4 tipos de errores en la lectura melódico ritmica}

Los errores más frecuentees aparecían en las medidas 8 y 9 contenidas en las células $\mathrm{C}$ y C', y consistían en duplicar los valores, de forma que dos corcheas se convertían en dos negras $(\zeta=J d)$, una blanca en una redonda $(\delta=0)$. Esto se daba en los tres niveles en los que se trabajó.

El encadenamiento con la medida siguiente demostró que determinados sujetos intentaban continuar su lectura al perder la pulsación, otros la continuaban a pesar de lo lento del fragmento.

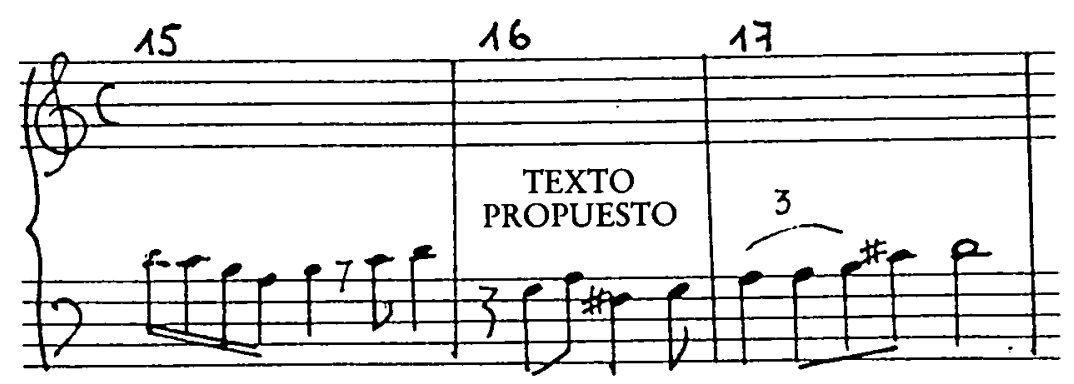


En la medida 12 se daban errores relacionados con el intento de establecer un valor largo en el tercer tiempo.

Los ejemplos siguientes, tomados de la lectura de las medidas 15,16 y 17 , ilustran las conductas de los sujetos.

El ejemplo 1 muestra que el sujeto no volvió sobre el error de la medida 15, del que no se sabe si era consciente o no. Invirtió los valores de los silencios, se equivocó en la alteración de la medida 16 después de haber reducido a la mitad el valor de la pausa, que se convirtió en una semi-pausa. La repetición positiva tuvo que hacerla sobre los dos elementos melódicos y rítmicos. La buena autocorrección le permitirá continuar la lectura.

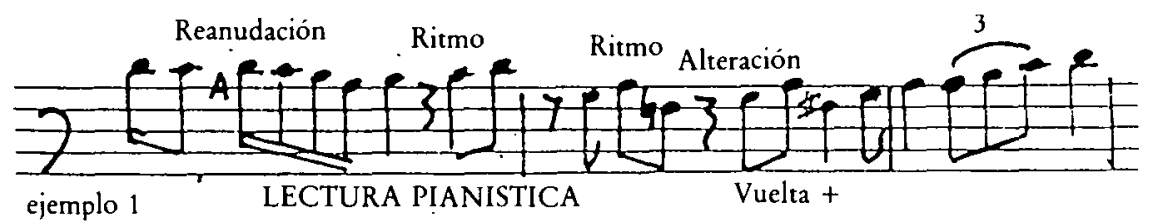

El ejemplo 2 muestra los intentos de otro sujeto de nivel P2 para salir del impás en el que a la lectura de los ritmos se añadia la dificultad de lectura de las notas en clave de fa. La colocación de las notas precedía a la del ritmo. Al contrario que en el ejemplo anterior, la alteración no apareció en esta lectura pianística y no fue objeto de una vuelta autocorrectora. La lectura de las dobles corcheas era insegura pero el ritmo que hay que darles estaba presente de alguna forma en la memoria del lector, cuyas reanudaciones muestran un intento de reajuste entre lo que había conseguido y lo que debería llegar a conseguir. Lo mismo ocurriría con la medida 16, que el sujeto intentó situar mediante un acompasado vocal del primer tiempo y la pronunciación del nombre de las notas.
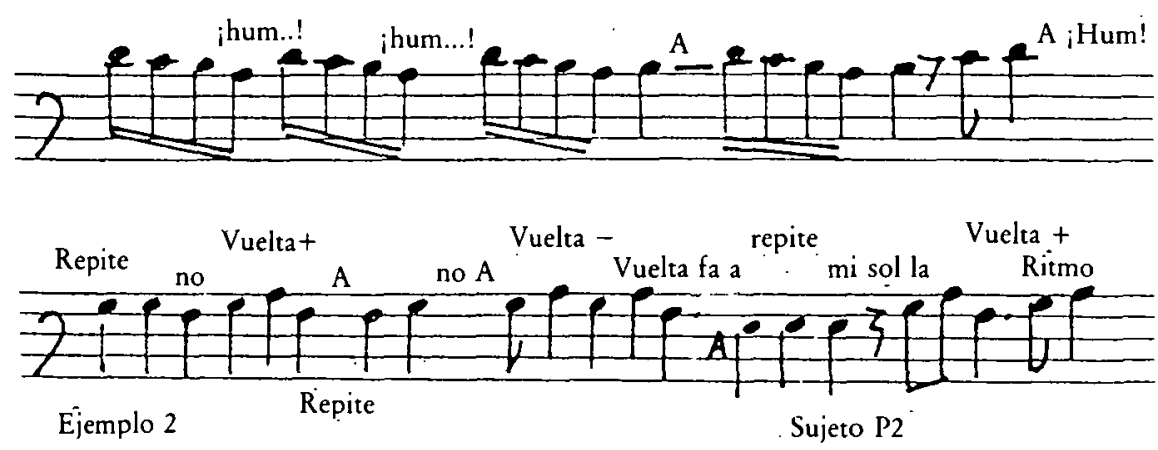

El ejemplo 3 nos ofrece un nivel inferior de lectura en un sujeto de EL2. La primera vuelta negativa sobre las corcheas de la medida 15 desestabilizó la 2:" parte de la lectura. La lectura resultaba difícil en los dos planos, melódico y rítmico. La aparición de un si bemol demuestra que el sujeto no se situaba claramente en el plano de la tonalidad mayor o menor del fragmento. Percibía de forma confusa que lo que estaba tocando no era lo que debería tocar, ya que évolvió atrás varias veces, pero fracasó en la autocorrección y perdió la pulsación rítmica. La lectura se hizo incomprensible.

En conjunto, el análisis de los distintos errores demuestra que si bien los ritmos normalmente estaban desorganizados en los sujetos de iniciación, resultaban más reconocibles en los de preparatorio. En el nivel elemental, a pesar de los errores, los mejores lectores conseguían mantener la pulsación rírmica hasta el final del fragmento. 

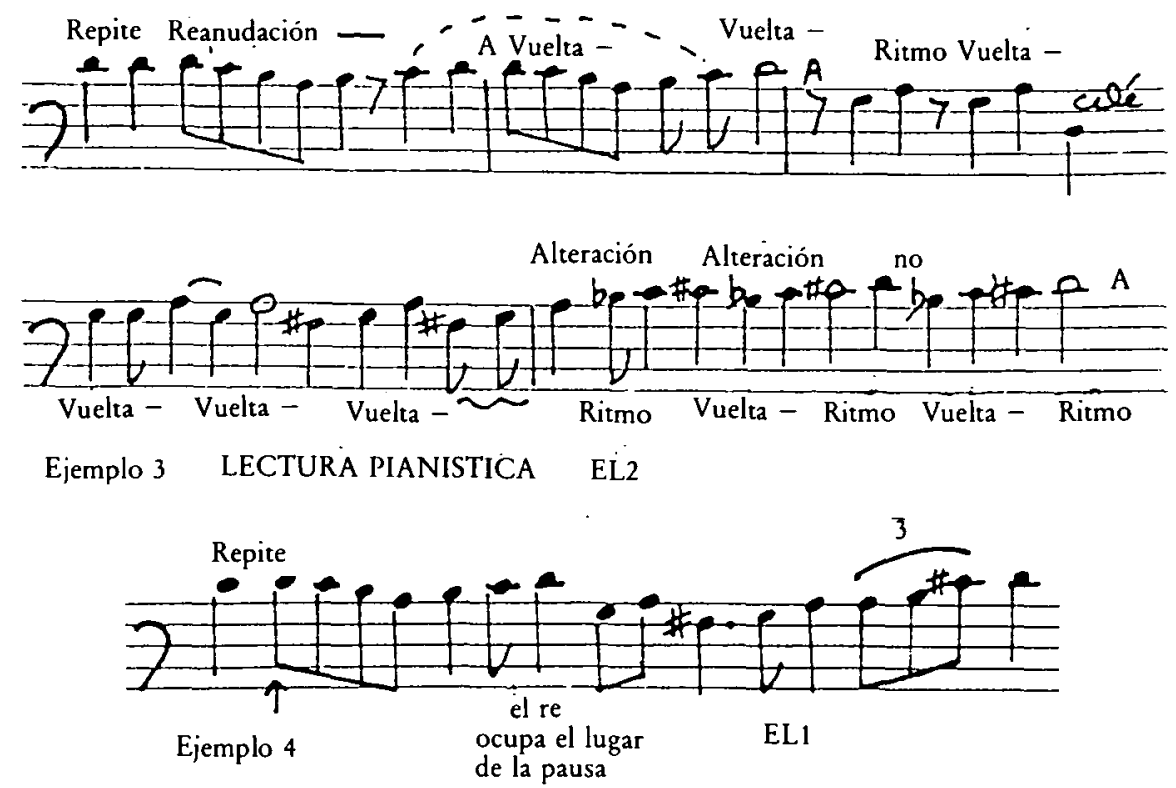

El ejemplo 4, tomado de la lectura de un sujeto del grupo elemental, muestra que éste se equivocó en el descifrado de las dobles corcheas y los silencios de las medidas 15 y 16 . A pesar de la repetición del primer re de la medida, el sujeto marcó su primer tiempo sobre el re del grupo de las corcheas: la conversión de las dobles corcheas en corcheas dio 5 tiempos a la medida. El re, quinto tiempo, pasó a ocupar el lugar de la pausa (primer tiempo de la medida 16). El sujeto mantuvo la pulsación hasta el final del fragmento. Además, la repetición del primer re (medida 15) estaba incluida en el tempo; el tiempo añadido a la medida 15 se quitó de la medida 17, donde el re «blanca» tomó el valor de una "negra». A pesar de estas modificaciones, el equilibrio de la lectura queda a salvo.

\section{TABLA I}

\begin{tabular}{llllllll}
\hline & \multicolumn{4}{c}{$\begin{array}{c}\text { Lectura de las alteraciones en la } \\
\text { prueba melódico-rítmica binaria al } \\
\text { piano }\end{array}$} \\
\cline { 2 - 7 } & D & D' & P1 & P2 & EL1 & EL2 \\
\hline Errores u olvido de las alteraciones & 1 & 6 & 12 & 5 & 1 & 10 \\
\hline
\end{tabular}

La forma en que se reparten las notas, su proximidad o separación, influyen en la percepción visual y la interpretación al piano. El ejemplo 5 muestra que la confusión de claves (cclaves) sirve para asegurar la continuidad creciente o decreciente de la gama, como ya lo habíamos encontrado en la lectura de las notas (Dubost, 1989).

La forma en que el lector lleva a cabo determinados agrupamientos, que le hacen la lectura más fácil, indica que los procesos perceptivos puestos en juego en la lectura visual de la música son compatibles con las leyes de proximidad y contigüidad de la "Gestaltheorie».

Según esta teoría, cuando el material desconcierta a la organización perceptiva, ésta tiende a restaurar una forma de conjunto más estable y fácil de aprehender. El lector no vacila en desplazar determinadas notas para restablecer una dirección de conjunto más conforme a un modelo familiar. 


\section{CONCLUSION}

\section{Sobre errores de lectura}

Los errores de exactitud de las notas, su designación en la partitura, su localización en el teclado, implican deformaciones melódicas o rítmicas, y se traducen en olvidos (alteraciones).

El error de designación puede significar que se ha tomado una clave por otra (confusión de clave) o que se ha dado a una nota un nombre distinto, el que tendría en clave de fa (error de nota).

Si se encuentra esta diferenciación en el plano vocal y en el plano pianístico, los dos tipos de errores se traducen en incorrecciones en la localización espacial: las notas no se tocan en las teclas adecuadas en los dos casos.

Desde el punto de vista perceptivo, nada nos permite decir si se trata realmente de errores debidos a una mala percepción de las claves como tales o un fallo en el teclado en el momento de la interpretación de las notas. Dicho de otra forma, los sujetos pueden haber percibido bien la nota y haberla tocado mal debido a una torpeza cuyas causas habría que determinar, o bien haberla percibido mal y haberla tocado mal como consecuencia de esa percepción incorrecta.

Hemos intentado controlar este aspecto comprobando si los errores se encontraban en los mismos lugares del texto en la lectura vocal y en la pianística en cada sujeto, pero la gran labilidad de los errores de una lectura a la otra no permite aclarar este hecho.

Al ver las notas, el sujeto se forma representaciones de ellas.

Si el olvido de la alteración se puede considerar como no respuesta al lugar en que las notas alteradas se deben interpretar al piano, no se puede decir si la nota, con su característica particular, se leyó realmente o simplemente fue objeto de un recuerdo sin memoria, si la información desapareció en el trayecto de su restitución, si era su designación lo que fue imposible recordar o, incluso, si es que la información que se identificó no dejó huellas, si la huella se borró o si permanecía pero no fue posible evocarla en la situación de test. No se puede decir si en el contexto concreto en el que el sujeto inscribe su experiencia, estas dificultades están relacionadas o no con causas emocionales causantes de perturbaciones de la atención, de distracción en la tarea cognitivo-motriz.

No se sabe tampoco qué sentido podria tener la tonalidad del texto propuesto, y si se ha percibido, cómo se ha comprendido.

Lo que se puede constatar es que en todos los niveles de aprendizaje se encuentran sujetos que se entregan a la tarea como si la mirada pasara más allá de las alteraciones accidentales o de la alteración que aparece en el armazón del fragmento (Dubost, 1989), pareciendo que éstas no atraen su atención.

\section{Sobre conductas de lectura}

En los descifrados propuestos, la indicación de tempo dada al comienzo de la prueba obligaba a los sujetos a leer lo mejor posible sin perder tiempo. En una tarea del tipo de una tarea de eficiencia (Huteau, 1985), en la que la consigna implícita que se exige es la de tener la rapidez requerida cometiendo el menor número de errores posible, el sujeto podrá decidir si 
mantiene el tempo en detrimento de la precisión o al revés. Los sujetos en situación de doble exigencia, que no están seguros de responder a los dos objetivos, se encuentran en una situación conflictiva. El conflicto impide al sujeto leer el texto correctamente y le lleva a cometer errores para resolverlo.

Nosotros mantenemos la idea de que en la lectura oral el error surge como compromiso entre lo que es el texto y lo que el lector se siente capaz de hacer con él (Bettelheim, 1983). De su actitud para salir de la situación peligrosa en la que se encuentra dependerá la continuación de la lectura, el interés que pueda seguir teniendo en ella.

Para permanecer vigilante y mantener la postura que supone la lectura visual, el lector establece una barrera entre su mundo interno y el de sus percepciones externas (Freud, 1915-1972), allí donde no desea dejarse distraer por aquello que perturbaría el estado de concentración necesario para realizar la tarea. Al relajarse la acción inhibidora surge el error, la dificultad: la nota falsa, el ritmo deformado, aparecen como imperativos de una discordancia a rectificar.

En este contexto de experiencias nada podría indicarnos si el sujeto que no corrige su error es realmente consciente o no de ello. Simplemente se podrá decir que algunos consiguen un reequilibrio más satisfactorio de su lectura mientras que otros no lo consiguen.

En el caso de la autocorrección, la memoria no será sólo de reconocimiento sino también evocadora, lo que facilitará la vuelta sobre el error, y ello permitirá al lector una seguridad en el dominio del tiempo y el espacio para continuar leyendo.

Los buenos lectores alcanzarán una mayor soltura, mientras que los novatos, que mantuvieron durante varias notas una altura incorrecta, fracasarán varias veces en sus intentos de autocorrección, como si no fueran capaces de llegar a un acuerdo entre el efecto obtenido y el efecto a obtener, al menos de forma inmediata.

\section{Sobre la práctica pianistica}

La organización del material musical en la partitura influye en la lectura. La presencia de elementos rítmicos la facilita si las estructuras rítmicas pueden ser aprehendidas y reconocidas por los sujetos, lo que no ocurre en el caso de los alumnos de iniciación, en especial en los repetidores que en nuestros experimentos al parecer se enfrentaban a unas dificultades de los textos que desbordaban su nivel de competencia.

Los buenos lectores de nuestra muestra se agrupaban en el nivel de los EL1, cuyas puntuaciones eran superiores a las de los de otros grupos. Por ello la desviación entre las adquisiciones conceptuales y su realización al piano era menor, mostrando una mejor coordinación viso-motriz que los otros. Leían con pocas dificultades y en el tempo exigido.

Sin embargo, los resultados observados en la lectura de las medidas 8 y 9 (células $C+C^{\prime}$ ) del texto demostraban que la primera lectura vocal hablada no suponía una ventaja real para la lectura pianística, pues, si bien esas células en esa posición eran conocidas y bien solfeadas en el.nivel elemental, eran erróneas en el poco virtuoso al piano, donde no es posible marcar con el gesto la división en cuatro de los tiempos en el espacio; si las 
dificultades se interiorizan se convierten en barreras mentales difíciles de superar en la continuidad, sobre todo después de los valores largos.

Esta observación habla en favor del entrenamiento en el descifrado pianístico de los ritmos, capaces no sólo de mitigar las diferencias entre lectura vocal e instrumental, sino también de mejorar la rapidez de la lectura. ¡Podrá la práctica corriente e intensiva del descifrado pianístico salir de la sombra en la que le ha sumido un trabajo centrado en exceso en la técnica para la interpretación!

\section{Notas}

${ }^{1}$ Comentarios sobre la forma de puntuación

Una nota puede ser el soporte de uno o varios errores. Si las categorias de confusión de clave y errores de notas no se recubren, nos podríamos haber encontrado con una confusión de clave o un error de nota y además se podría haber añadido un error de alteración, de ritmo o de registro; por eso en un primer tiempo contabilizamos cada una en su categoría.

Los errores de registro, que sólo aparecen en el instrumento, se contabilizaron por sepa-. rado, con el fin de preservar el análisis comparado de los resultados en las modalidades de lectura, vocal e instrumental.

Esta forma de puntuación nos permitió explorar con precisión los distintos tipos de errores posibles.

En cada lector se hizo un reagrupamiento según las tres categorías: exactitud, dificultades y tiempo. En la lectura melódico-ritmica, cuyos resultados comentamos, tuvimos en cuenta las dos variables, melódica y rítmica, considerando la exactitud de células cortas y contabilizando un solo error cuando son inexactas una de las dos variables. Las células aisladas no tenian notas comunes a las dos. logía.

La puntuación de trabas representaba la suma de todas las categorías presentadas en su tipo-

La puntuación de tiempos presentaba, en número de segundos, la suma de los tiempos de la lectura.

Dificultades rítmicas en la lectura musical visual durante los primeros años del aprendizaje del piano.

\section{B. Dubost.}

$$
\text { CLE, 1991, 10, 101-115. }
$$

Datos sobre la autora: B. Dubost, psicóloga, doctorada en tercer ciclo de Psicología (1989, París X-Nanterre), Primer premio de piano (CNM de Mans).

Artículo original: Presentation des difficultés Rythmiques dans la lecture a vue musicale au cours des premières années d'apprentisage pianistique. Les Sciences de l'Education, 1990, 3-4, 109-129. Reproducido con autorización. Traducción de Inés Marichalar.

(C) De todos los artículos. Deberá solicitarse por escrito autorización de CLE para el uso en forma de facsímil, fotocopia o cualquier otro medio de reproducción. CLE se reserva el derecho de interponer las acciones legales necesarias en aquellos casos en que se contravenga la ley de derechos de autor. 